\title{
The Asakura-Oosawa model in the protein limit: the role of many-body interactions
}

\author{
A. Moncho-Jordá and A.A. Louis, ${ }^{1}$ P.G. Bolhuis, ${ }^{2}$ and R. Roth ${ }^{3}$ \\ ${ }^{1}$ Department of Chemistry, Lensfield Rd, Cambridge CB2 1EW, UK \\ ${ }^{2}$ Department of Chemical Engineering, University of Amsterdam, \\ Nieuwe Achtergracht 166, NL-1018 WV Amsterdam, Netherlands \\ ${ }^{3}$ Max-Planck Institut für Metallforschung, Heisenbergstrasse 3, \\ D-70569 Stuttgart, Germany and ITAP, University of Stuttgart, \\ Pfaffenwaldring 57, D-70569 Stuttgart, Germany
}

\begin{abstract}
We study the Asakura-Oosawa model in the "protein limit", where the penetrable sphere radius $R_{A O}$ is much greater than the hard sphere radius $R_{c}$. The phase behaviour and structure calculated with a full-many body treatment show important qualitative differences when compared to a description based on pair potentials alone. The overall effect of the many-body interactions is repulsive.
\end{abstract}

\section{INTRODUCTION}

The Asakura Oosawa (AO) model [1], also known as the penetrable hard sphere model 2], was first introduced almost 50 years ago to describe depletion effects in colloidpolymer mixtures. The colloids are modelled as hard spheres (HS) of radius $R_{c}$, and the "polymers", or AO particles, as penetrable hard spheres (PHS), which interact as ideal particles with each other, but as HS of radius $R_{A O}$ with respect to the colloids. In spite of its simplicity, this model has been instrumental in understanding the phase-behaviour of polymer-colloid mixtures. For example, increasing the polymer concentration can lead to a fluid-fluid or fluid-solid demixing of the colloidal particles. The origin of this phase transition arises from the depletion effect, which is easily illustrated by calculating the free-energy of two HS in a bath of PHS AO particles. Each HS excludes a volume $\frac{4}{3} \pi\left(R_{c}+R_{A O}\right)^{3}$ from the PHS particles, but when two HS approach, their exclusion volumes overlap, resulting in more free volume available for the PHS spheres. This translates into an effective depletion pair potential between the two particles of the form [1, 2]

$$
\beta V_{A O}(r)=-z_{A O} \frac{4 \pi}{3}\left(\sigma_{c p}\right)^{3}\left\{1-\frac{3}{4} \frac{r}{\sigma_{c p}}+\frac{1}{16}\left(\frac{r}{\sigma_{c p}}\right)^{3}\right\},
$$

in the range $\sigma_{c}<r \leq 2 \sigma_{c p}$, where $\sigma_{c p}=R_{A O}+R_{c}$ and $z_{A O}$ is the fugacity of the AO PHS particles. The $\mathrm{AO}$ potential is always attractive, with a well-depth that increases with the fugacity (or number density) of AO particles. Simple geometrical arguments show that for size ratios $q=R_{A O} / R_{c} \leq 0.1547$ there are no higher order many-body interactions beyond the effective pair potential. For larger $q$, however, multiple overlap of the depletion zones can occur, leading to many-body interactions. As with many soft matter systems, these are often difficult to calculate, and so a common approximation is to ignore them, and treat a system in the effective pair-potential approximation [3, 4]. For the AO model, this works surprisingly well for descriptions of phase behaviour up to size-ratios of $q \approx 1[\underline{5},[]]$.
In this paper we study the AO model for $q=$ $R_{A O} / R_{c}>>1$. For polymer-colloid systems this is often called the nano-particle or protein limit, because small particles such as proteins are needed to achieve the large size-ratios. Clearly, a pair potential picture should break down for large enough $q$, where many-body effects are expected to dominate. For ideal polymers in the limit $q<1$, the effect of many-body interaction in polymer and colloid mixture has been studied by Meijer and Frenkel [5], who found that these interactions stabilise the liquid phase. Recent work has shown that for $q>>1$, many-body effects can qualitatively affect the phase behaviour [7, [8, [9].

The AO model was originally developed for size-ratios $q<1$, where $R_{A O}$ taken to be the radius of gyration $R_{g}$ of ideal polymers 1, 21. For $q>>1$ this simple mapping no longer holds, although the AO model can still be mapped onto a model for ideal polymers by correctly defining an effective $R_{A O}$ radius [8]. However, our goal here is not so much to study colloid-polymer mixtures, but rather to investigate the effect of many-body interactions on a well-defined system. The AO model has the particular advantage that the effective pair interaction $V_{A O}(r)$ is exactly known. By directly calculating the phase behaviour and structure of a two-component AO model, and comparing it to an effective one-component model with the AO pair potential, we can systematically study the effect of many-body interactions. The insight gained from this well characterised system should increase our appreciation of the complexity of many-body effects in soft matter systems.

The paper is organised as follows: In section 2 we calculate the phase-behaviour of the AO model, using Monte Carlo (MC) simulations and several simple theories. In section 3 we describe the pair correlation functions, and also the effective colloid-colloid structure factors. Finally, we discuss our results in section 4 . 


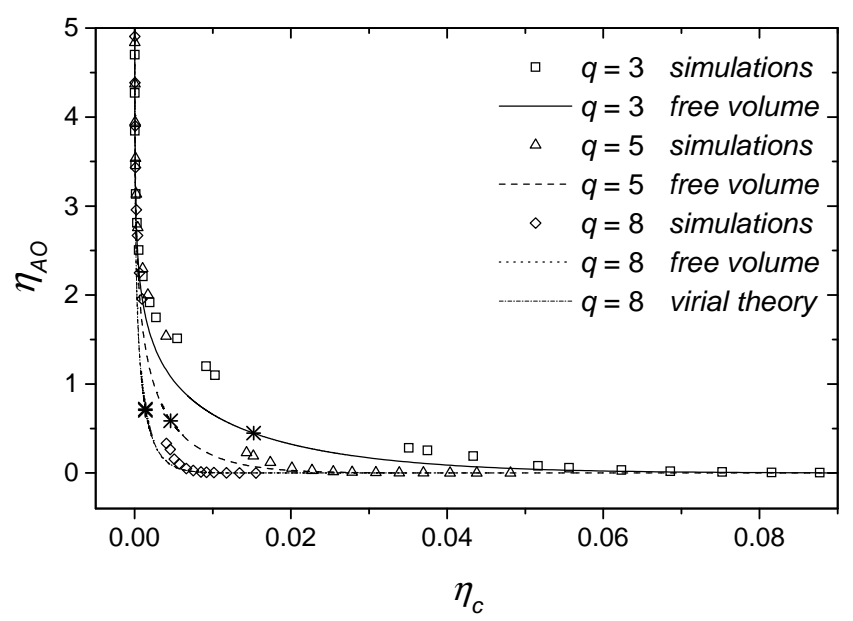

FIG. 1: Fluid-fluid binodals for size-ratios $q=3,5,8$. Plotted are the absolute packing fractions $\eta_{A O}=\frac{4}{3} \pi \rho_{A O} R_{A O}^{3}$ and $\eta_{c}=\frac{4}{3} \pi \rho_{c} R_{c}^{3}$. Also shown are the binodals calculated with the free volume theory of ref. [13] and their corresponding critical points (asterisks). Note that the critical colloid packing fraction tends to zero with increasing $q$. The free volume and virial theory binodals for $q=8$ can not be distinguished since both theories converge in the limit of large $q$.

\section{PHASE BEHAVIOUR}

\section{A. Monte Carlo Simulations}

We performed Gibbs ensemble Monte Carlo 10 simulations in the semi-grand ensemble, where colloids are treated canonically and the PHS in the grand canonical ensemble, for three size ratios $q=3,5,8$. The total number of colloidal particles $(N=108)$ is hence fixed, although they can exchange boxes. The chemical potential or fugacity of the PHS is kept constant by the usual grand canonical MC insertion and extraction moves 10|. Further details of the method can be found in refs. 11, 12]. This setup is equivalent to an $\mathrm{AO}$ mixture in osmotic equilibrium with a reservoir of only PHS particles 13 .

Results for the binodals are shown in Fig. 1 For increasing $q$, the critical colloid packing fraction $\eta_{c}=$ $\frac{4}{3} \pi \rho_{c} R_{c}^{3}$ tends to zero, while the PHS packing fraction $\eta_{A O}=\frac{4}{3} \pi \rho_{A O} R_{A O}^{3}$ increases. It is also instructive to compare the binodals on a log-log plot, shown in Fig. 2 which emphasises parts of the binodals further from the critical point. For example, we see that the binodals cross at very low $\eta_{c}$.

It is often convenient to plot the phase diagrams in the semi-grand ensemble, where the colloids are treated in the canonical ensemble and the PHS in the grand canonical ensemble. This is equivalent to setting up an AO mixture in osmotic equilibrium with a reservoir of only PHS particles 13]. Because the PHS particles are ideal, the fugacity $z_{A O}=\exp \left(\beta \mu_{A O}\right)=\rho_{A O}^{r}$. The results in this representation are shown in Fig. 3

The binodals in Figs. 1 - 3 are quite different to those

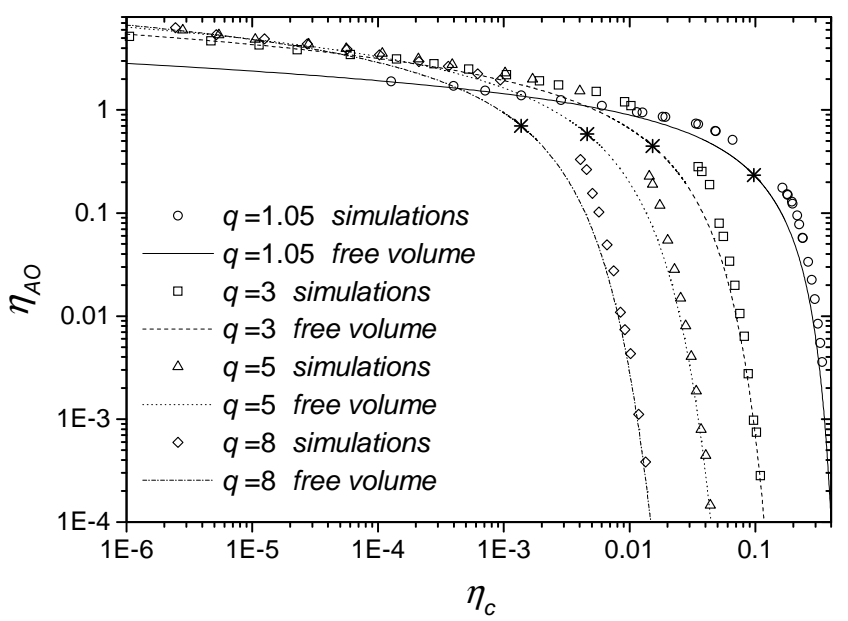

FIG. 2: Same as in Fig. 1 but on a log-log scale. This emphasizes a different part of the binodals, showing that the relative agreement of the free-volume theory with the simulations is about the same for all size-ratios. Again, the critical points of the free volume binodals are shown as asterisks. Here we also include results for $q=1.05$, taken from ref. [12].

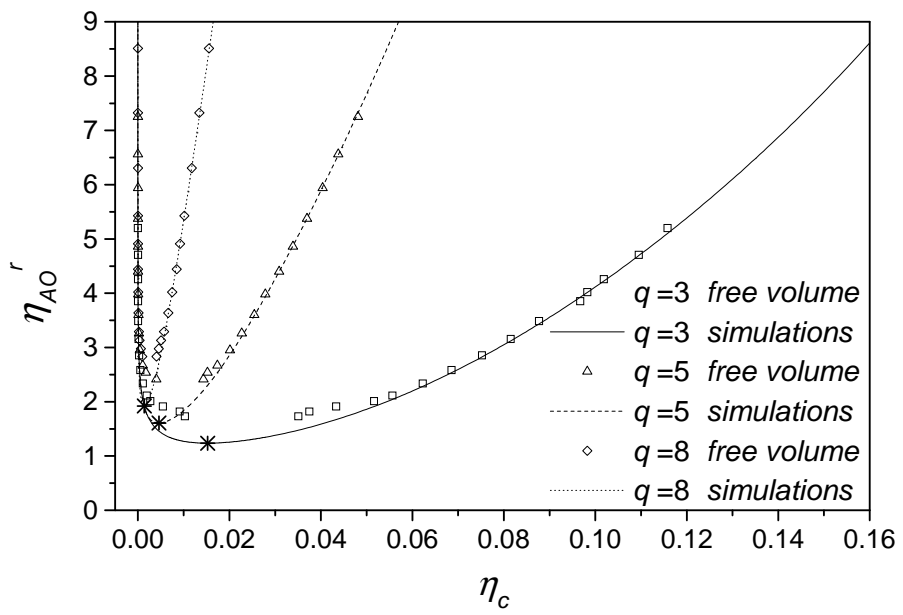

FIG. 3: Fluid-fluid binodals for size-ratios $q=3,5,8$. The $\mathrm{x}-$ axis denotes the colloid packing fraction $\eta_{c}$ and the y-axis the packing fraction $\eta_{A O}^{r}=\frac{4}{3} \pi \rho_{A O}^{r} R_{A O}^{3}$ of a reservoir of pure PHS particles at the same chemical potential as the two-component AO system. Also shown are binodals calculated with the free volume theory of ref. [13], which agree very well with the simulations.

of the AO model for $q<1$, shown, for example, in refs. 6, 13]. First of all, they are at much lower packing fractions of the colloids. In fact, the critical colloid packing fraction tends to zero for increasing $q$. Moreover, in the reservoir representation, the binodals are much narrower, an effect that becomes more pronounced with increasing $q$. Both effects are similar to what has been found for mixtures of ideal polymers and colloids [ $[$, , 8].

The physical origin of the low critical colloid packing fraction is the large cross-interaction between the two dif- 
ferent species. Perhaps a simpler way of viewing this is to first imagine a binary HS mixture of particles of equal diameter $\sigma_{c}$, but with a cross diameter $\sigma_{12}>\sigma_{c}$. As $\sigma_{12}$ increases, the system will phase separate at lower and lower volume fractions of the HS particles. A phase-diagram like that shown in Fig. 1 1 would be symmetric in the packing fractions of the two species. However, one could also choose to represent the packing fraction of one of the two species, say species 1 , as $\eta_{1}=\frac{1}{6} \pi \rho_{1} \sigma_{12}^{3}$. For large enough $q$ the phase diagram would then closely resemble that of Fig. [1 since the packing fraction of species 1 would be so low that it could be replaced by an AO PHS particle without significantly affecting the phase behaviour. This way of obtaining the large $q$ limit of the AO model also suggests that a simple virial theory should become increasingly accurate as $q$ increases and the critical packing fractions decrease. The next section will show that such a virial theory works very well indeed 14].

\section{B. Two-component free volume and virial theories}

A general Helmholtz free-energy for the twocomponent AO model can be written as:

$\frac{F\left(N_{c}, N_{A O}, V\right)}{V}=f=f_{c}^{H S}\left(\rho_{c}\right)+f_{A O}^{i d}\left(\rho_{p}\right)+f_{c-A O}\left(\rho_{c}, \rho_{A O}\right)$

where the colloids are treated as hard-spheres, and the AO PHS as ideal particles. We suppress the dependence on temperature $T$, since our model is athermal.

Whereas $f_{c}^{H S}\left(\rho_{c}\right)$ and $f_{A O}^{i d}\left(\rho_{A O}\right)$ are well understood [17], less is known about the $f_{c-A O}\left(\rho_{c}, \rho_{A O}\right)$ term. In the free-volume theory of Lekkerkerker et al. [13], $f_{c-A O}$ is approximated as

$$
f_{c-A O}^{\text {free }}=\rho_{A O} \omega\left(\eta_{c}, q\right) .
$$

In other words, terms proportional to $\rho_{A O}^{2}$ and higher in $f_{c-A O}$ are ignored. $\omega\left(\eta_{c}, q\right)$ can then be interpreted as the free energy of inserting a single AO PHS particle into a bath of HS colloids at packing fraction $\eta_{c}$. In their classic paper, Lekkerkerker et al. [13] calculated $\omega\left(\eta_{c}, q\right)$ from scaled particle theory:

$$
\begin{aligned}
\omega\left(\eta_{c}, q\right) & =-\ln \left(1-\eta_{c}\right)+A \frac{\eta_{c}}{1+\eta_{c}} \\
& +B\left(\frac{\eta_{c}}{1+\eta_{c}}\right)^{2}+C\left(\frac{\eta_{c}}{1+\eta_{c}}\right)^{3},
\end{aligned}
$$

where $A=3 q+3 q^{2}+q^{3}, B=\frac{9}{2} q^{2}+3 q^{3}$, and $C=3 q^{3}$. In the nomenclature of ref. [13], $\omega\left(\eta_{c}, q\right)=-\ln \left(\alpha\left(\eta_{c}, q\right)\right)$ with $\alpha\left(\eta_{c}, q\right)$ the so-called free-volume fraction, which defines the particle packing fraction $\eta_{A O}^{r}$ in a reservoir at the same chemical potential as the mixture i.e. $\eta_{A O}=\alpha\left(\eta_{c}, q\right) \eta_{A O}^{r}$. As $q$ increases, so does the relative strength of the cross interaction, leading to lower and lower critical colloid packing fractions. It therefore
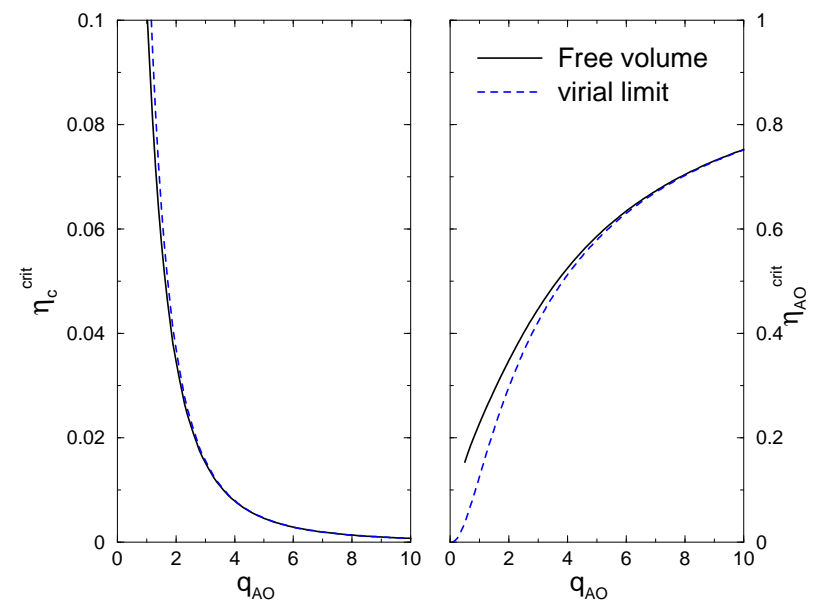

FIG. 4: The limiting behaviour of the critical point, given by Eqs. (6) and (7), is compared to the full free-volume theory [13] calculations. The differences rapidly decrease with increasing $q$.

makes sense to expand $f_{c-A O}^{f r e e}$ in powers of $\eta_{c}$ :

$$
f_{c-A O}^{\text {free }}=\rho_{A O}\left(\eta_{c}(1+q)^{3}+\mathcal{O}\left(\eta_{c}^{2}\right)\right) .
$$

The leading term is proportional to the second crossvirial coefficient. In other words, for low $\eta_{c}$ (and for any $\left.\rho_{A O}\right)$, free-volume theory reduces, as expected, to a simple virial theory.

For the virial theory defined by Eq. (2) and the leading term of Eq. (5), the large $q$ limit of the critical points can be derived:

$$
\begin{aligned}
\lim _{q \rightarrow \infty} \eta_{c}^{\text {crit }} & =\frac{1}{(1+q)^{3}} \sim \frac{1}{q^{3}} \\
\lim _{q \rightarrow \infty} \eta_{A O}^{\text {crit }} & =\frac{q^{3}}{(1+q)^{3}} \sim 1 \\
\lim _{q \rightarrow \infty} \eta_{A O}^{r, c r i t} & =\frac{\exp (1) q^{3}}{(1+q)^{3}} \sim \exp (1) .
\end{aligned}
$$

Since $\eta_{c}^{\text {crit }} \rightarrow 0$ as $q \rightarrow \infty$, it follows that free-volume theory shows the same limiting behaviour, which is demonstrated in Fig. [4

A comparison with Figs. 1-3 shows that, even though higher order $\rho_{A O}$ effects in $f_{c-A O}$ are ignored, freevolume theory agrees remarkably well with simulations, just as was found earlier for $q \approx 1[12,18]$. The main deviations are found near the critical point; these can partially be ascribed to the fact that free-volume theory is a mean-field theory, with the wrong critical exponents etc..., which normally leads to more rounded binodals. Nevertheless, the simulations are quite consistent with the limiting behaviour for the critical points derived in Eqs. (6) - (8).

In Fig. 1] we also show the binodal for $q=8$ obtained from the simple cross-virial theory. The differences with free-volume theory are not visible on the scale of the 
graph. For smaller $q$ the virial theory is not quite as good as the free-volume theory, but it still provides a semi-quantitative description of the binodals, suggesting that the basic physics can be understood at this simpler level.

The good agreement between simulations and freevolume theory for the fluid-fluid binodals suggests that we can use the latter to estimate the position of the triple point. This occurs when the gas, liquid and solid are in equilibrium, and an easy way to estimate the location of the triple point is to set up an equilibrium between the gas phase branch of the binodal, and a HS fluid at the freezing transition. This results in an approximate position for the triple point at $\eta_{c}=0.494$ and:

$$
\eta_{\text {AO }}^{r, \text { triple }} \approx \frac{\pi}{6} \beta P_{\text {coex }} \sigma_{c}^{3} q^{3} \approx 6.12 q^{3},
$$

where the reduced coexistence pressure of a HS fluid at freezing, $\beta P_{\text {coex }} \sigma_{c}^{3}$, is known from simulations $[10]$. In the limit of large $q$ it is virtually impossible to fit any PHS spheres into the colloidal crystal, while the gas-phase binodals are at extremely low $\eta_{c}$; we therefore expect this relationship to become asymptotically exact for large size ratios. In fact, Eq. (9) gives a good prediction for the free-volume triple point for all size ratios where a triple point exist, i.e. even for $q<1$. For example, at $q=0.8$ free volume theory gives $\eta_{A O}^{r}=3.13$ while Eq. (9) would predict $\eta_{A O}^{r}=3.17$. This analysis shows that, in the semi-grand ensemble, the triple point moves to extremely large values of $\eta_{A O}^{r}$, compared to the critical point.

\section{One-component theory with pair potentials}

It is instructive to compare the results for the twocomponent AO model with those of an effective onecomponent model. As mentioned in the introduction, the AO PHS particles can be integrated out to derive an exact pair potential, valid for all $q$, and given by Eq. (1). For $q \leq 0.1547$ this leads to an exact description of the system, but for larger $q$, many-body interactions must be invoked. Nevertheless, for practical reasons, the pair approximation is often used in soft matter physics. In many situations this works well, but here we expect it to break down as $q$ increases.

For large $q$, the pair potential of Eq. (11) becomes very long-ranged with respect to the colloidal diameter $\sigma_{c}$. Therefore mean-field theory, for which the free-energy takes the form

$$
\frac{F^{M F}\left(N_{c}, z_{A O}, V\right)}{V}=f_{c}^{H S}\left(\rho_{c}\right)+\frac{1}{2} \rho_{c}^{2} \int d \mathbf{r} V_{A O}(r) \text {. }
$$

should become asymptotically exact. For this simple "van der Waals limit", the critical colloid packing fraction is always given by $\eta_{c}^{\text {crit }} \approx 0.13$, independent of potential details. Here we work in the semi-grand ensemble, where the effective pair potential picture has a consistent statistical mechanical interpretation [6, 19]. In Fig. 5, the bin-

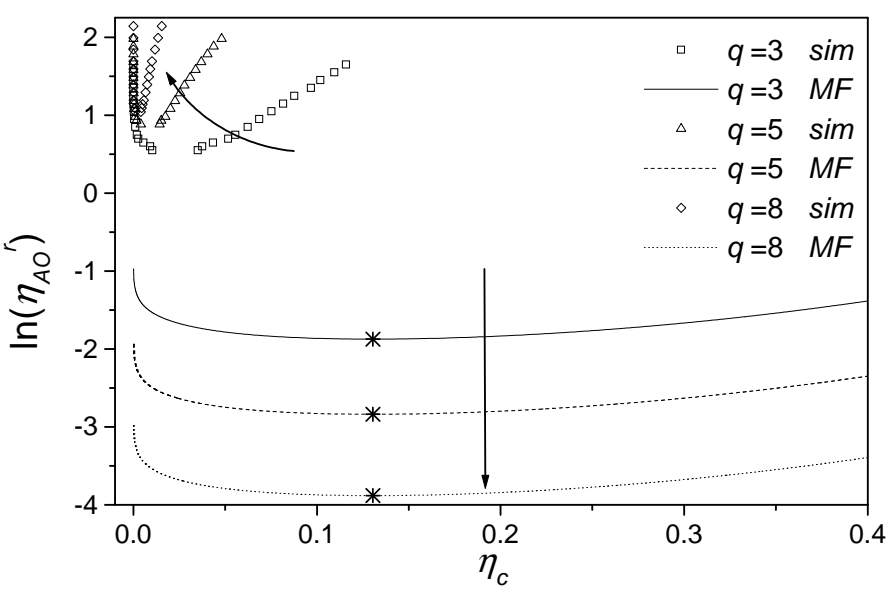

FIG. 5: A comparison of the binodals for the two-component AO model, calculated by GEMC simulations, with an effective one-component picture, calculated with the pair potential $V_{A O}(r)$ and Eq. (10). Note the qualitative differences in the limit of large $q$ : For the one-component picture $\eta_{c}^{\text {crit }}$ tends to 0.13 , and $\eta_{A O}^{r, \text { crit }}$ tends to 0 , whereas the full two-component picture shows the opposite behaviour: $\eta_{c}^{\text {crit }}$ tends to zero, and $\eta_{A O}^{r, c r i t}$ tends to a constant. These trends are depicted by the arrows. The differences are due to the effects of many-body interactions.

odals from Eq. (10) are compared to the full 2-component simulations.

Besides the obvious quantitative differences for the location of the critical points (note that the y-axis is logarithmic!), there are important qualitative differences as well. For example, the two-component binodals are much more narrow. Furthermore, the critical points show opposite scaling behaviour with increasing size ratio $q$ : For the two-component model $\eta_{A O}^{r, c r i t}$, tends to a constant and $\eta_{c}$ tends to zero, whereas for the one-component model $\eta_{A O}^{r, c r i t}$ tends to zero and $\eta_{c}$ tends to a constant.

The dominant effect of the many-body interactions appears to be repulsive, since phase-separation occurs at a much higher packing fraction of the AO PHS particles. In fact, the three-body interactions have already been calculated for the AO model [20]; they are repulsive for all geometries. This would seem consistent with the overall effect of the many-body interactions. However, this interpretation is most likely too naive. For example, we expect that the fourth order term is attractive again, and that the series oscillates, as was recently found in simulations of a self avoiding walk polymer system[21]. In general, the sign of a many-body interaction can vary in a complex way with coordinates. A good example is given by the 3-body HS depletion interactions calculated in ref. 20]. Furthermore, it has recently been shown that the effects of many-body interactions in a mixture of interacting polymers and colloids are attractive [8, 22], the opposite of what we find for the AO model. These examples suggest that it is generally quite difficult to make simple predictions regarding the effect of many-body in- 
teractions on phase-behaviour.

\section{PAIR STRUCTURE}

\section{A. Radial distribution functions}

Given the unusual phase-behaviour of the AO model in the protein limit, it should be interesting investigate the effect of many-body interactions on the pair structure. To that end we performed Monte Carlo simulations of the colloid-colloid and colloid-AO pair correlations. Representative examples are shown in Fig. 6] Both $g_{c c}(r)$ and $g_{c-A O}(r)$ have fairly weak structure, with only one main peak. The reasons for this are two fold: 1) The colloid packing fraction is very low. 2) More generally, long ranged interactions lead to less sharply peaked radial distribution functions [3]. We also compared the simulations to pair correlations derived from the test-particle route for a recently developed fundamental measure theory (FMT) density functional theory (DFT) [23]. This DFT shows the same phase-behaviour as the free volume theory, which we have shown to be very accurate for the AO model. We therefore expect the DFT to be rather good in this limit, although correlations are often a more sensitive probe of a DFT than phase behaviour is. Results are compared in Fig. [6 to the simulations. For lower $\eta_{A O}$ the agreement is very good, but for higher $\eta_{A O}$ some deviations are found. This may partially be because the state point for the highest $\eta_{A O},\left(\eta_{c}=0.004, \eta_{A O}=0.6\right)$ is very close to the free-volume critical point (which lies at $\left.\eta_{c}=0.0046, \eta_{A O}=0.586\right)$, while the critical point for the simulations is further away.

On might expect that, due to the low colloid densities, the correlation functions should be rather straightforward to calculate with integral equation methods [17]. In Fig. 7] we compare, for one state-point, some representative results from the Percus Yevick (PY) and Hypernetted Chain (HNC) approximations 17]. PY systematically underestimates the peaks, a general effect that becomes more pronounced with increasing $\eta_{A O}$, and which is similar to what happens for binary HS mixtures [3]. HNC appears to be more accurate, but it suffers from a nonsolution line, a mathematical artifact where no solutions are found. This non-solution line occurs well before the expected spinodal, and makes HNC less useful. Finally we note that direct functional differentiation of the FMT DFT in the bulk leads to PY correlation functions. The test-particle route we apply here is thought to generally be more reliable.

\section{B. Structure factors}

For scattering experiments, a more useful measure of the pair correlations is given by the colloid-colloid structure factor $S(k)$. These are shown in Fig. 8 for some of
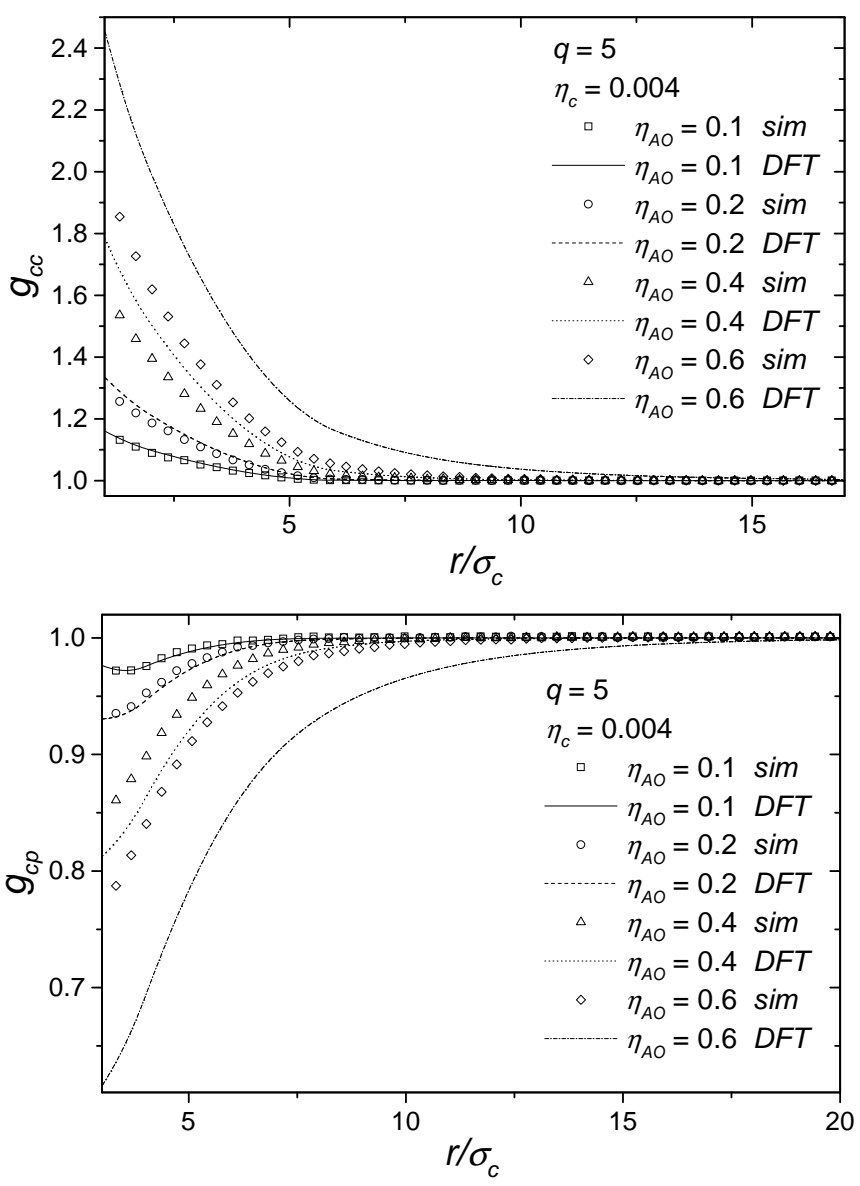

FIG. 6: The colloid-colloid and colloid-PHS radial distribution functions for $q=5$ at several state-points. We compare direct simulations with results from the test-particle route of a fundamental measure theory DFT 23]. For lower packing fractions the agreement is good, but when the DFT binodal is approached, differences become larger.

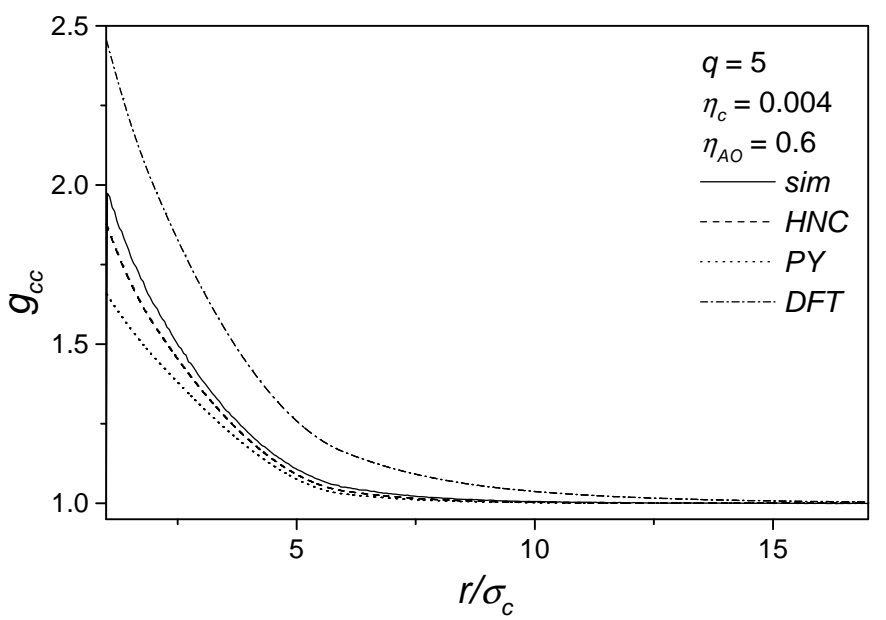

FIG. 7: A comparison between DFT, HNC, and PY approaches to the colloid-colloid structure, and results from simulations. 


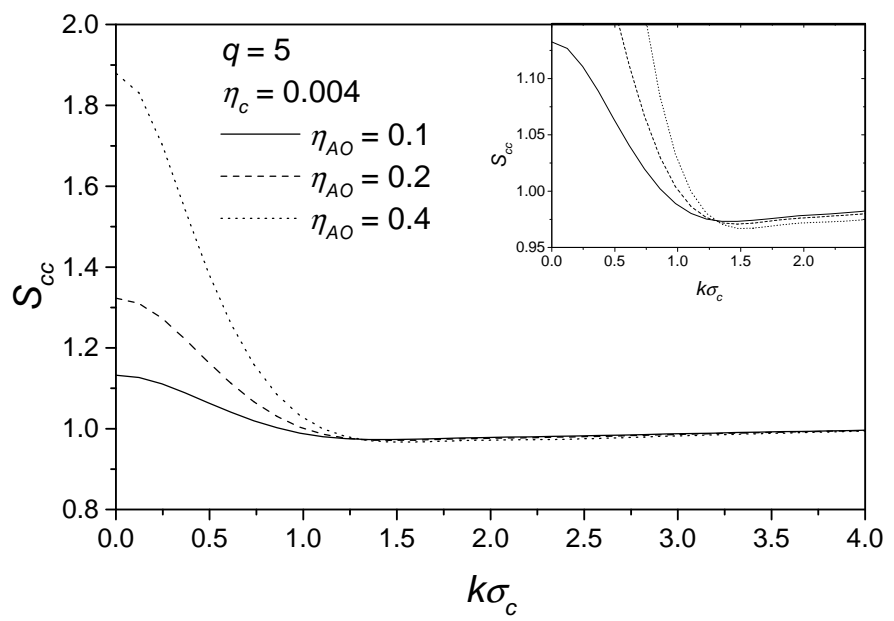

FIG. 8: Structure factors from simulations for some of the same state points as Fig. 6] Inset: the first isosbestic point where the structure factor is invariant to changes in $\eta_{A O}$.

the same state points as in Fig. [6 The $S(k)$ shows virtually no structure, except for a maximum at $S(0)$. This behaviour seems to be generic for the large $q$ limit of the AO model. Another interesting observation is the occurrence of an isosbestic point $k_{i s o s}$, a value of $k$ where $S(k)$ is invariant to changes in $\eta_{A O}$. A recent theory 24 for isosbestic points predicts that $k_{i s o s} \sigma_{c}$ decreases with increasing range 25. The theory predicts that $k_{i s o s} \sigma_{c} \approx \pi$ for very short ranged potentials, and that for the $\mathrm{AO}$ model it can be approximated as:

$$
k_{i s o s} \sigma_{c} \approx \pi /\left(1+0.42 q / 2+(0.42 q)^{2} / 12\right)
$$

Even though this theory was derived for the small $q$ limit of a one-component model, it still appears to be semiquantitative here, since we find an isosbestic point at $k_{\text {isos }}=1.3 \pm 0.05$ and Eq. (11) would predict $k_{\text {isos }}=1.30$. For other $q$ 's we also found good agreement. This further suggests that one experimental signature of the longranged nature of the AO model is the late upturn of $S(k)$ toward a maximum at $S(0)$. This is illustrated in Fig. 9] We note that in a recent paper, Tuinier and Brulet [26] have shown similar behaviour from a one-component calculation with only effective pair potentials. They also performed small angle neutron scattering experiments on lysozyme-polysaccharide mixtures, measuring structure factors $S(k)$ that appear quite similar in shape to those predicted here for the AO model. Since these authors obtained similar qualitative behaviour to our simulations from a one-component theory, the qualitative behaviour of $S(k)$ that we observe is most likely caused by the longranged nature of the pair potentials, and not so much by the many-body character of the interactions.

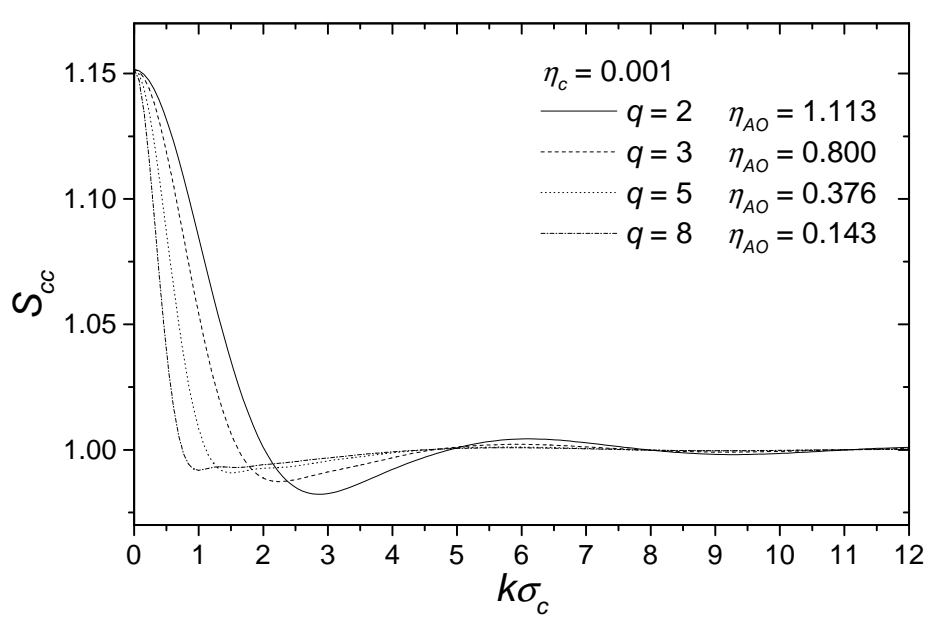

FIG. 9: Structure factors from HNC for different values of $q$. The state points are chosen to give a similar value of $S(0)$. The main effect of increasing $q$ is to decrease the value of $k \sigma_{c}$ where $S(k)$ begins to increase towards its maximum at $S(0)$.

\section{Effective colloid-colloid pair potentials}

The extremely low packing fractions of $\eta_{c}$ studied here might suggest that one could approximate the pair correlation function by its zero density limit:

$$
\lim _{\eta_{c} \rightarrow 0} g_{c c}(r)=\exp \left[-\beta V_{A O}(r)\right] .
$$

However, this is not correct, as suggested by the large differences between the binodals calculated only with $V_{A O}(r)$ and those calculated for the full two-component AO model. We demonstrate this explicitly in Fig. 10. where $g_{c c}(r)$ at the same $\eta_{A O}^{r}$ (which means the same $V_{A O}(r)$ ), but for different $\eta_{c}$ was calculated with the FMT DFT approach. At $\eta_{c}=0$, eq. (12) is of course exactly obeyed, but this no longer holds for the other values of $\eta_{c}$. The effect of the many-body interactions lowers the peak of $g(r)$, which is consistent with the behaviour of the binodals, where the effect of the manybody interactions is to reduce the cohesion between the HS colloids.

A general theorem states that for any $g(r)$ and density $\rho$, there exists a unique pair potential $v_{g}(r ; \rho)$ which will reproduce that $g(r)$, regardless of the underlying manybody interactions [27]. We inverted the $g_{c c}(r)$ at two state-points with HNC and PY inversions. The results are shown in Fig. 11. The two inversion methods give very similar results (especially at low polymer packing fractions, where both inversions can not be distinguished on the scale of the graph), suggesting that the potential obtained is indeed close to the true $v_{g}\left(r ; \rho_{c}\right)$. Note that $v_{g}\left(r ; \rho_{c}\right)$ is different from the simple potential of mean force $-\ln \left(g_{c c}(r)\right)$, which shows that correlation effects are important here. As expected, $v_{g}\left(r ; \rho_{c}\right)$ is less attractive than the bare pair potential $V_{A O}(r)$. It is also slightly longer ranged. If there were no many-body forces, then 


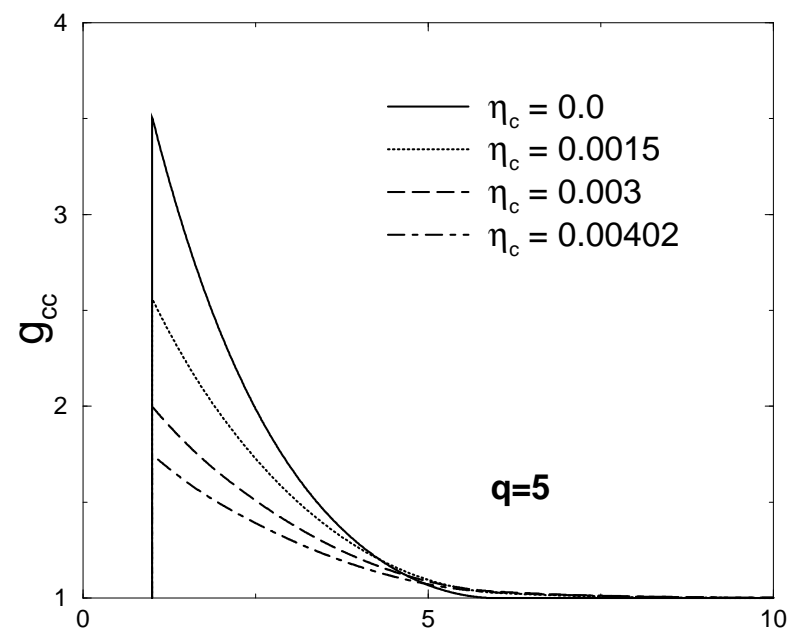

FIG. 10: Radial distribution functions for systems with different $\eta_{c}$. For each $\eta_{c}$ the reservoir packing fraction was $\eta_{A O}^{r}=$ 0.964792 , which corresponds to $\eta_{A O}=0.4$ at $\eta_{c}=0.00402$.

$v_{g}\left(r ; \rho_{c}\right)$ would be equal to $V_{A O}(r)$ at all densities. The differences can therefore be attributed to many-body interactions, whose overall effect is to weaken the effective pair potential.

At each density one could use $v_{g}(r ; \rho)$ to extract the colloid-colloid pair correlations, and also the osmotic pressure through the compressibility route. However, in general a large density dependence of the effective pair potential also implies difficulties like the representability issues discussed in ref. [19], making a pairwise description less useful.

\section{Effective PHS-PHS pair potentials}

In the limit of large $q$ it might seem tempting to try the opposite of the usual Asakura-Oosawa strategy, and integrate out the HS colloids instead of the PHS particles. This can easily be done, and the potential between two isolated PHS particles takes the same functional form as in Eq. (1), but with colloid and PHS AO parameters switched. One difference is that $V_{A O}(r)$ is now relevant down to $r=0$, because there is no HS repulsion to restrict it to $r \geq \sigma_{c}$, as is the case for the colloids. However, a description based only on the effective pair potential immediately leads to problems. The lack of hard-core repulsion means that the effective one-component system of PHS particles with an AO type pairwise attraction falls into the class of catastrophic potentials defined by Ruelle 28], for which there is no thermodynamic limit. This does not mean that one cannot derive a consistent thermodynamics by integrating out the HS colloids. Rather, because the PHS particles can overlap so easily, many-body effects are always very important, especially at small $r$, and are necessary to stabilise the effective PHS system. A similar situation was recently found when integrating out internal degrees of freedom for a solution of
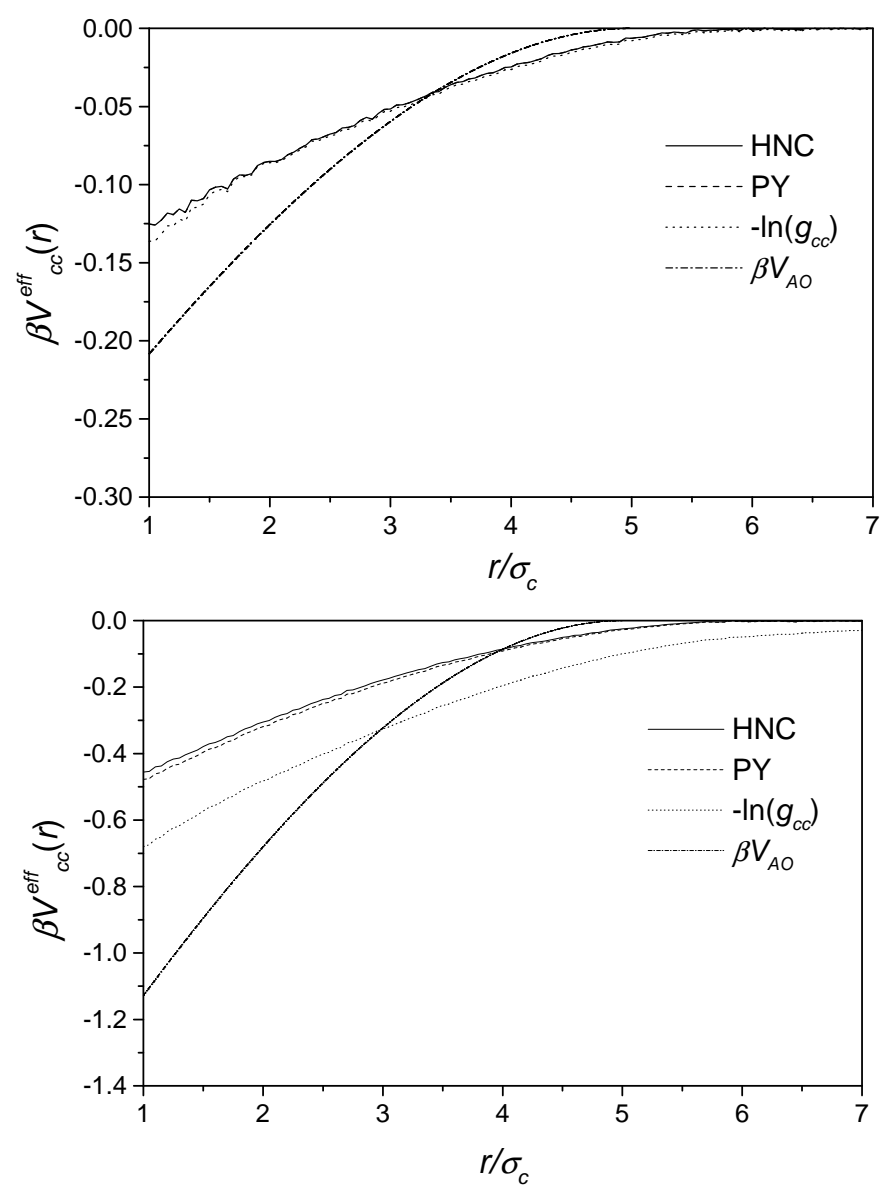

FIG. 11: Inversions of the colloid-colloid pair correlation functions for two state-points: up: $\eta_{c}=0.0402, \eta_{A O}=0.1$ and down: $\eta_{c}=0.0402, \eta_{A O}=0.6$. For each we show the results of a PY and an HNC inversion, as well as the potential of mean force, $-\ln \left(g_{c c}(r)\right)$ and the bare two-body potential $V_{A O}(r)$. HNC and PY are indistinguishable for the lower polymer packing fraction (upper figure).

polymers in a poor solvent 29], as well as for a Gaussian Core model [30]. Both examples can lead to catastrophic pair potentials, even though the underlying many-body system is stable.

\section{CONCLUSIONS}

In conclusion then, we have shown that in the so-called protein limit, where $q>>1$, the behaviour of the twocomponent $\mathrm{AO}$ model differs significantly from a description based on an effective pair potential description alone. We summarise our main results:

- In the limit of large $q$, the $\mathrm{AO}$ binodals move to lower and lower colloid packing fractions $\eta_{c}$, and at the critical point, the $\mathrm{AO}$ particle packing fraction $\eta_{A O}$ tends to a constant. This is opposite to what is found from a pair potential description alone, and 
so this phase behaviour can be ascribed to manybody interactions.

- The free-volume theory of Lekkerkerker et al. 13. works remarkably well for the phase-behaviour. It reduces to a simple virial theory in the large $q$ limit, allowing us to extract limiting values for the critical points which are consistent with the simulations.

- The colloid-colloid pair-correlation functions show very weak structure. Even at very low packing fractions $\eta_{c}, g_{c c}(r)$ is not well described by its zerodensity limit $\exp \left[-\beta V_{A O}(r)\right]$. The pair correlations are well described by a recent fundamental measure theory density functional theory [23], at least if one is not close to the critical point.

- The overall effect of the many-body interactions is repulsive, as seen in the phase behaviour, and also in the structure.

- There are clear signatures of the long-ranged interactions in the structure factors $S(k)$. In particular, the value of $k \sigma_{c}$ where $S(k)$ begins to rise to its maximum at $k=0$, decreases with increasing $q$.

- A description based on effective pair potentials between the PHS particles, derived by integrating out the smaller HS colloids, leads to catastrophic systems with no thermodynamic limit.
Our aim in this paper was to study the effects of manybody interactions in a well-defined model system. Although it would be tempting to extract some more general insights about the role of many-body interactions in soft matter systems, this is not so easy to do. On the one hand, we can make predictions about the behaviour of a related many-body system, namely a mixture of ideal polymers and HS colloids in the limit of large $q[7,8]$ where we expect some similar trends. But on the other hand, if the ideal polymers are replaced by interacting ones, the behaviour changes: for example, the critical colloid packing fraction is almost constant, and the overall effect of the many-body interactions is attractive instead of repulsive 8]. It is clearly not always easy to predict the effect of the many-body interactions a-priori. We conclude from this that coarse-graining a soft-matter system to a representation where many-body interactions are important when compared to the pair interaction may not always be a very fruitful way forward. Sometimes it may be easier to treat the original system without this coarse-graining step.

\section{Acknowledgements}

A. Moncho-Jordá thanks the Ramón Areces Foundation (Madrid), and A.A. Louis thanks the Royal Society (London) for financial support.
[1] Asakura S, \& Oosawa F 1958 J. Polym. Sci. Polym. Symp. 33183.

[2] Vrij A 1976 Pure Appl. Chem. 48471.

[3] Louis A A 2001 Phil. Proc. Roy. Soc. A 359939.

[4] Likos C N 2001 Phys. Rep. 348267.

[5] Meijer E J and Frenkel D 1994 J. Chem. Phys. 1006873.

[6] Dijkstra M, Brader J M and Evans R 1999 J. Phys.: Condens. Matter 1110079.

[7] Sear R P 2001 Phys. Rev. Lett. 864696.

[8] Bolhuis P G, Meijer E J, and Louis A A 2003 Phys. Rev. Lett. 90068304.

[9] These models for ideal polymers resemble the system studied here, and some preliminary results were reported in ref. 8].

[10] Frenkel D and Smit B 2002 Understanding molecular simulations, 2nd ed. (Academic Press, San Diego).

[11] Bolhuis P G and Frenkel D 1994 J. Chem. Phys. 100 9869 (1994).

[12] Bolhuis P G, Louis A A and Hansen J-P 2002 Phys. Rev. Lett. 89128302.

[13] Lekkerkerker H N W, Poon W C K, Pusey P N, Stroobants A and Warren P B 1992 Europhys. Lett. 20 559 .

[14] If one were to keep $R_{A O}$ fixed, and then take the limit $q \rightarrow \infty$, then the so-called Widom-Rowlinson model 15] results, for which a virial theory also works very well [16]

[15] Widom B and Rowlinson J S 1970 J. Chem. Phys. 52 1670; we thank M. Schmidt for pointing out this connec- tion to us.

[16] Schmidt M 2001 Phys. Rev. E 63010101.

[17] Hansen J-P and McDonald I R 1986 Theory of Simple Liquids, 2nd Ed. (Academic Press: London)

[18] Dijkstra M and van Roij R 2002 Phys. Rev. Lett. 89 208303.

[19] Louis A A 2002 J. Phys.: Condens. Matter 149187.

[20] Goulding D and Melchionna S 2001 Phys. Rev. E 64 011403.

[21] Bolhuis P G, Louis A A and Hansen J-P 2001 Phys. Rev. E 64021801.

[22] Rotenberg B, Dzubiella J, Hansen J-P and Louis A A, cond-mat/0305533

[23] Schmidt M, Löwen H, Brader J M and Evans R 2000, Phys. Rev. Lett. 851934.

[24] Louis A A, cond-mat/0212073

[25] We note that independently of 24], R. Tunier and G.A. Vliegenthart have made similar predictions for isosbestic points.

[26] Tuinier R and Brulet A 2003 Biomacromolecules 428.

[27] Henderson R L 1974 Phys. Lett. A. 49 197; Chayes J T and Chayes L 1984 J. Stat. Phys. 36471.

[28] Ruelle D 1969, Statistical Mechanics: Rigorous Results, (W.A. Benjamin, Inc, London).

[29] Krakoviack V, Hansen J P and Louis A A 2003, Phys. Rev. E 67041801.

[30] Archer A J, Evans R and Roth R 2002, Europhys. Lett. 59 526; Archer A J and Evans R 2003, J. Chem. Phys. 
1189726. 